\title{
Surface Plastic Deformation by Sliding Elliptical Cylinder
}

\author{
Rostislav I. Nepershin \\ Plastic Deformation Systems Department, Moscow State University of Technology “STANKIN", Moscow, Russia \\ Email: nepershin ri@rambler.ru
}

Received November 2014

\begin{abstract}
Steady state plastic flow of the ideal plastic half-space surface by sliding elliptical cylinder is numerically calculated with account of contact friction effect. Numerical solution of the plane strain hyperbolic differential equations with unknown contact pressure distribution is treated as nonlinear vector equation for the steady state plastic flow condition. Pronounced effect of the ellipse boundary curvature on the plastic flow mode is shown. Engineering application of the computer model is surface plastic deformation technology to improve wear and fatigue resistance of metal parts.
\end{abstract}

\section{Keywords}

\section{Surface Layer, Plastic Flow, Ideal Plasticity, Sliding; Elliptical Cylinder, Friction}

\section{Introduction}

Surface plastic deformation by rigid tools is used in engineering technology to increase wear and fatigue resistance of metal contact parts. Specification of technology parameters for surface plastic deformation is difficult engineering problem induced by complex elastic-plastic deformation of the surface layer.

Finite element analysis of rigid cylinder rolling along elastic-plastic half space is considered in [1] [2]. But large plastic deformation compared with the elastic one, unknown plastic region boundaries, stress and velocity singularities at the intersection of the toll contact with surface boundary are key problems for accuracy of the finite element elastic-plastic analysis.

Plastic deformation in surface layer, deems, is dominant factor of the surface plastic technology, and models on the base of ideal plasticity theory [3] [4] is reasonable. Steady state ideal plastic flow induced by rolling of rigid cylinder is considered in [5] using a small parameter approach. Approximate analysis related with the rolling friction is given in [6]. Steady state plastic flow of surface layer induced by rolling and sliding of circular cylinder is investigated in [7]-[9].

Present steady state plastic flow model of surface layer is developed for rigid elliptical cylinder sliding with contact friction consideration. Variable curvature and ellipse orientation relative the surface boundary are effective features of the plastic flow mode. Sliding of the wedge [10] and circular cylinder [7]-[9] along the plastic surface are limit cases of the present model. 


\section{Problem Formulation}

Steady state plastic flow of an ideal plastic half-space surface layer induced by sliding of loaded long elliptical cylinder is considered. Plane strain plastic flow on the plane $x, y$ orthogonal to the cylinder axis is assumed with half-space moving velocity $V$ and nonmoving cylinder.

Stresses and velocities in the plastic region, loads and torque applied to the cylinder are non-dimension, using material plastic constant $2 k$ and sliding velocity $V$ as reference units. Elliptical section of the cylinder and scheme of the plastic region are shown in Figure 1.

Steady state plastic region $O A B$ and cylinder are non- moving in coordinates $x, y$ with the origin $O$ at the lower contact point of the cylinder with half-space boundary. First principal axis $x_{1}$ of the ellipse is inclined at the angle $\theta_{0}$ to the axis $x$. Minimal half-length of the ellipse axis is assumed as unit reference length, and ellipse form is defined by non-dimensional maximal half-length axis $a$. Ellipse boundary with the center $C$ can be written in parametric form in principal ellipse coordinates $x_{1}, x_{2}$

$$
x_{1}=a \cos \omega, y_{1}=\sin \omega,-\pi / 2 \leq \omega \leq \pi / 2
$$

Tangent angle $\theta$ to the axis $x_{1}$ at the point $x_{1}, x_{2}$ is defined as follows

$$
\operatorname{tg} \theta=-(\operatorname{ctg} \omega) / a
$$

Coordinates $x, y$ of the ellipse point $x_{1}, x_{2}$ are defined by shift and rotation transformation of the exses

$$
\begin{aligned}
& x=x_{C}+x_{1} \cos \theta_{O}-y_{1} \sin \theta_{O} \\
& y=y_{C}-x_{1} \sin \theta_{O}+y_{1} \cos \theta_{O}
\end{aligned}
$$

Angle $\theta_{O}$ at the point $O$ is defined from Equation (2) by specified parameter $\omega_{O}$. Coordinates $x_{C}, y_{C}$ of the ellipse center $C$ are found from Equations (1) and (3) with specified $\omega_{O}$ using the condition $x=y=0$

$$
\begin{aligned}
& x_{C}=\sin \omega_{O} \sin \theta_{O}-a \cos \omega_{O} \cos \theta_{O}, \\
& y_{C}=a \cos \omega_{O} \sin \theta_{O}-\sin \omega_{O} \cos \theta_{O} .
\end{aligned}
$$

Contact boundary $O A$ is defined by specified parameter $\omega_{A}$ for the point $A$. Coordinates $x, y$ of the boundary $O A$ with the tangent angle $\alpha$ to the axis $x$

$$
\alpha=\theta-\theta_{O}, \omega_{O} \leq \omega \leq \omega_{A}
$$

are defined by Equations (1)-(3).

Parameters $\omega_{O}, \omega_{A}$ and $a$ define ellipse form, the angle $\theta_{O}$ and contact boundary $O A$ on different segments of the ellipse. Length of the contact boundary $l_{c}$ is defined by integral

$$
l_{c}=\int_{\omega_{0}}^{\omega_{A}}\left[\cos ^{2} \omega+a^{2} \sin ^{2} \omega\right]^{1 / 2} d \omega
$$

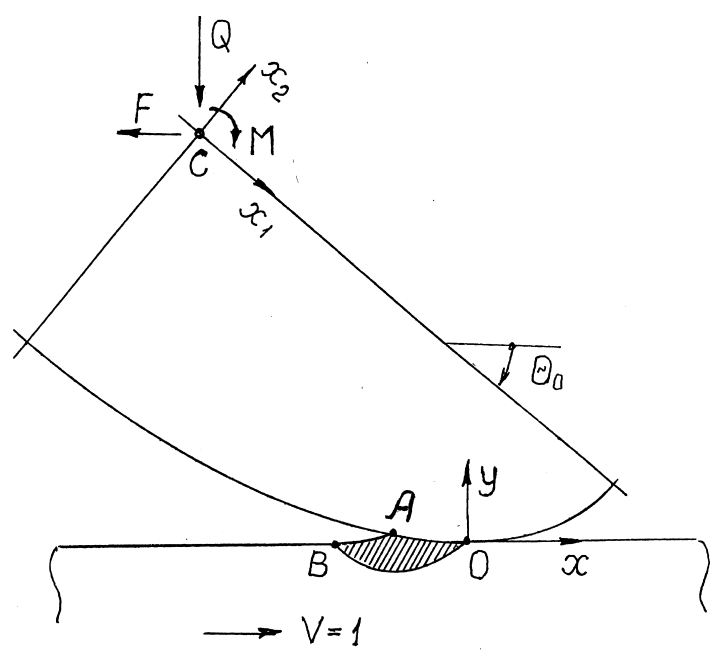

Figure 1. Steady state surface plastic region. 
Boundary $O A$ with contact pressure and shear stress define loads $Q, F$ and torque $M$ (Figure 1) using static equilibrium of the cylinder.

Plane strain plastic flow of the ideal plastic material [3] [4] is defined by hyperbolic differential Equations with orthogonal $\xi$ and $\eta$ slip lines

$$
\mathrm{d} x / \mathrm{d} y=\operatorname{tg} \varphi \text { for } \xi, \mathrm{d} x / \mathrm{d} y=-\operatorname{ctg} \varphi \text { for } \eta,
$$

Henky's Equations for the mean stress $\sigma$ and angle $\varphi$

$$
\mathrm{d} \sigma-\mathrm{d} \varphi=0 \text { along } \xi, \mathrm{d} \sigma+\mathrm{d} \varphi=0 \text { along } \eta
$$

where $2 k=1$ is used as unit stress, and Geiringer's Equations for the velocity projections $V_{\xi}, V_{\eta}$ on the slip lines

$$
\mathrm{d} V_{\xi}-V_{\eta} \mathrm{d} \varphi=0 \text { along } \xi, \mathrm{d} V_{\eta}+V_{\xi} \mathrm{d} \varphi=0 \text { along } \eta
$$

Stress and velocity components in coordinates $x, y$ are related with $\sigma, \varphi, V_{\xi}$ and $V_{\eta}$ variables by Equations

$$
\sigma_{x}=\sigma-0.5 \sin 2 \varphi, \sigma_{y}=\sigma+0.5 \sin 2 \varphi, \tau_{x y}=0.5 \cos 2 \varphi
$$

and

$$
V_{x}=V_{\xi} \cos \varphi-V_{\eta} \sin \varphi, V_{y}=V_{\xi} \sin \varphi+V_{\eta} \cos \varphi
$$

\section{Boundary Conditions}

Stress free boundary $A B$ (Figure 2) is the stream line of the steady state plastic flow, with the principal stresses $\sigma_{1}=0, \sigma_{2}=-1$ along this boundary, and we have stress and velocity boundary conditions on $A B$

$$
\sigma=-0.5, V_{\xi}+V_{\eta}=0
$$

Friction on the boundary $A O$ is specified by contact shear stress $0 \leq \tau_{c} \leq 0.5$, used in technological plasticity problems with large contact pressures [11]. Incline angle $\gamma$ for the $\eta$ slip line on the contact boundary (Figure 2(a)) is found from third Equation (10) using specified $\tau_{c}$ value

$$
\gamma=0.5 \cos ^{-1}\left(2 \tau_{c}\right)
$$

Angle $\varphi$ between the $\xi$ slip lines and axis $x$ on the boundary $A O$ with the tangent angles $\alpha$ is defined as

$$
\varphi=\pi / 2+\gamma-\alpha, 0 \leq \alpha \leq \alpha_{A}
$$

Zero normal component of the velocity and the angle $\gamma$ define boundary condition for the velocities $V_{\xi}$ and $V_{\eta}$ on the boundary $A O$

$$
V_{\xi}-V_{\eta} \operatorname{tg} \gamma=0
$$

Half-space velocities $V_{x}=1, V_{y}=0$ are continues on the rigid-plastic boundary $O B$, and from Equations (11) it follows

$$
V_{\xi}=\cos \varphi, V_{\eta}=-\sin \varphi
$$

Mean stress $\sigma_{O}$ at the point $O$ is defined from second Equation (8) for the $\eta$ slip line, using first boundary condition (12) with $\varphi=\pi / 4$ at the point $B$, and Equation (14) with $\alpha=0$ at the point $O$

$$
\sigma_{O}=-(0.5+\gamma+\pi / 4)
$$

Fun angle $\psi$ for the $\xi$ slip lines at the singular point $A$ with tangent angle $\beta$ on the stress free boundary $A B$ is found using Equation (14) with $\alpha=\alpha_{A}$

$$
\psi=\pi / 4+\gamma-\left(\alpha_{A}+\beta\right)
$$

Angle $\beta$ is defined during problem solution using condition $y=0$ at the point $B$.

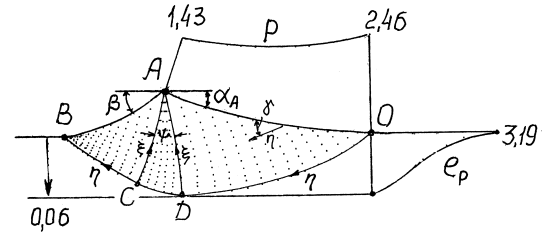

(a)

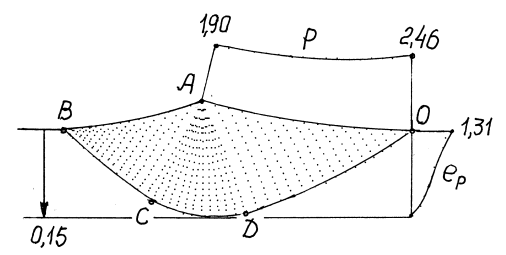

(b)

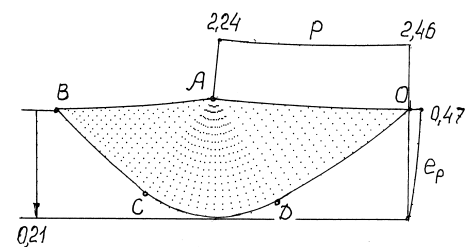

(c)

Figure 2. Plastic regions with pressure and plastic. Strain distributions for variants (a) 1; (b) 2 and (c) 3 in Table 1. 
Limit value $\alpha_{A}^{*}$ for the steady state plastic flow is found from Equation (18) with $\psi=0$

$$
\alpha^{*}{ }_{A}=\pi / 4+\gamma-\beta
$$

Corresponding limit parameter $\omega^{*}$ and contact length $l_{c}$ are found from Equations (5) and (6).

\section{Numerical Solution}

Numerical solution of the problem with specified parameters $a, \omega_{O}, \omega_{A}$ and $\tau_{c}$ begins by initial guess for the angle $\beta$ with constrain $\alpha_{A}<\alpha^{*}$ in Equation (19).

Then mean stress $\sigma_{A}$ at the singular point $A$ on the contact boundary $A O$ is found from boundary condition (12) and second Equation (8) for the $\eta$ slip line

$$
\sigma_{A}=-(0.5+\psi)
$$

Fan angle $\psi$ is defined by Equation (18) with the angle $\alpha_{A}$ found from Equations (2) and (5) for specified parameters $a, \omega_{O}$ and $\omega_{A}$.

Initial mean stress distribution $\sigma_{i}$ is specified at the nodes $i=1,2,3, \ldots, N$ on the contact boundary $A O$ proportional the angles $\alpha_{i}$, defined by the parameters $\omega_{i}$ in the interval $\omega_{O} \leq \omega_{i} \leq \omega_{A}$. Mean stress $\sigma_{i}$ distribution, boundary condition (14) for the angles $\varphi_{i}$ and contact boundary $A O$, defined by Equations (1) and (3), specify Cauchy's boundary value problem for differential Equations (7) and (8).

Cauchy's problem is solved using numerical procedures [11] with the result of slip lines with $\sigma, \varphi$ variables calculation in the region AOD (Figure 2(a)). Then $\sigma$ and $\varphi$ variables are specified at the singular point $A$ using second Equation (8), followed by numerical solution of Riemann's problem in the region $A D C$ with known $\sigma, \varphi$ variables on the slip line $A D$. Finally, inverse Cauchy's problem is solved numerically in the region $A C B$, using known $\sigma, \varphi$ variables on the slip line $A C$ with $\sigma=-0.5$ on calculated stress free boundary $A B$. Then initial guess for the angle $\beta$ is corrected to satisfy condition $y=0$ at the point $B$.

So, slip lines in the plastic region are calculated for specified stress $\sigma$ distribution on the contact boundary AO. Next procedure is numerical calculation of the velocity field in the plastic region using conditions (15) on the boundary $A O$ and (16) on the boundary $O B$. First, velocities $V_{\xi}, V_{\eta}$ are calculated in the region $A O D$ by numerical solution of the mixed boundary value problem for Equations (9) with known $\varphi$ angles at the slip line nodes. Then velocity field is calculated in the region $A D C$ from Riemann's problem with known $V_{\xi}, V_{\eta}$ on the slip lines $A D$ and $C D$, followed by velocity field calculation in the region $A C B$, using known $V_{\xi}, V_{\eta}$ on the slip lines $A C$ and $C B$, with the result of $V_{\xi}$ and $V_{\eta}$ calculation on the stress free boundary $A B$.

Velocities $V_{\xi}$ and $V_{\eta}$ must satisfy steady state plastic flow condition in Equation (12). If we define specified $\sigma_{\boldsymbol{i}}$ values on the boundary $A O$ as the vector $\boldsymbol{\sigma}$ in $N$ dimension space and values

$$
f_{i}=\left(V_{\xi}+V_{\eta}\right)_{i}
$$

at the $N$ nodes of the boundary $A B$ as the vector function $f$ in the same $N$ dimension space, then we have nonlinear vector Equation (22) for the steady state plastic flow problem solution

$$
f(\sigma)=0
$$

because numerical procedures for calculation of the slip lines and velocity field in the plastic region give unique relation between vectors $\sigma$ and $f$. Equation (22) with $N \leq 20$ was solved using numerical procedures [12] for Broyden's method [13].

Equation (22) is solved with accuracy $\max f_{i} \leq 10^{-3}$.

Then contact pressure distribution

$$
p_{i}=-\left(\sigma_{i}-0.5 \sin 2 \gamma\right)
$$

was calculated on the boundary $A O$, followed by integration of pressure and contact shear stresses to find loads $Q, F$ and torque $M$ (Figure 1).

\section{Numerical Results}

For numerical solution of the problem FORTRAN program was written with input parameters $a, \tau_{c}, \omega_{O}, \omega_{A}$ and output data in the form of tables and graphical mapping of the slip line nodes as pixels on the monitor screen. Stream lines in the plastic region with accumulated plastic strain $e_{p}$ and orthogonal grid distortions were calculated using numerical procedures in [14]. 
Numerical examples below were calculated for the ellipse parameter $a=2$, three variants of the pairs $\left\{\omega_{O}, \omega_{A}\right\}$ and three typical values $\tau_{c}$ for practical surface plastic deformation technology to illustrate variable contact curvature and friction effects.

Three variants of the ellipse contact boundary $A O$, defined by the parameters $\omega_{O}$ and $\omega_{A}$, are given in Table $\mathbf{1}$. Corresponding ellipse incline angle $\theta_{O}$, tangent angle $\alpha_{A}$ at the point $A$, ellipse center $C$ and contact length $l_{C}$ are calculated from Equations (2), (4), (5) and (6). In variants 1 to 3 the incline angles $\theta_{O}$ and contact angles $\alpha_{A}$ are decreased with decrease of the ellipse curvature and increase of the contact length $l_{c}$.

Calculated slip lines in the plastic region with distributions of contact pressure $p$ and accumulated plastic strain $e_{p}$ of the material at the exit from the plastic region with shear contact stress $\tau_{c}=0.1$ are shown in Figure 2 for the ellipse boundary variants in Table 1. Curvature decrease of the ellipse contact boundary results in more homogeneous contact pressure distribution and decrease of the gradient and value of the plastic strain distributions through thickness of the surface plastic layer. But mean contact pressure is increased, and for smooth boundary with $\tau_{c}=0$ it is approached to Prandtl's flat punch value $p=1+\pi / 2$.

Velocity hodographs on the plane $V_{x}, V_{y}$ in the plastic regions in Figure 2 are presented in Figure $\mathbf{3}$ for the same three variants of the ellipse contact boundary (Table 1) and shear contact stress $\tau_{c}=0.1$. Velocities $V_{x}, V_{y}$ are calculated using Equations (11) with known variables $V_{\xi}, V_{\eta}, \varphi$ at the slip line nodes and mapped by pixels on the monitor screen.

Unit velocity vector at the point $B$ is decreased along the stress free boundary $A B$ with tangent angle increase to the $\beta$ at the point $A$. Then velocity vector direction is rotated at the singular point $A$ to the tangent angle $\alpha_{A}$ at the contact boundary, followed by increase of the vector module tangent to the boundary $A O$ from the point $A$ to the point $O$ where it is unit horizontal vector.

Boundary $B C D O$ of the plastic region on the plane $x, y$ (Figure 2) is mapped to the point $B C D O$ on the hodograph plane (Figure 3), as the result of the velocity continuity with moving half-space given by Equations (16).

Curvature decrease of the ellipse boundary AO from variant 1 to 3 in Table 1, and from (a) to (c) in Figure 2 and Figure 3 results in drastic velocity field non-homogeneous decrease, with homogeneous velocity field $V_{x}=$ $1, V_{y}=0$ for the flat sliding Prandtl's punch.

Initial orthogonal grid distortions with the stream lines for the plastic regions in Figure $\mathbf{2}$ are shown in Figure 4 for the same variants of the ellipse contact boundaries in Table 1. Plastic boundary $O B$ with the half-space is shown by dash line.

Movement of material point along the stream lines is calculated using steady state velocity fields in Figure 3 for constant horizontal displacement increment $d x$ at the entry to the plastic regions.

Displacement of the material point along the stream lines in the plastic region is calculated using mean velocity vectors in the "time" interval $d x$, because the half-space velocity is unit.

Final displacement $\Delta x$ is defined by the slow material point, moving on the path $B A O$, while the fast material point is moved with unit velocity on horizontal path tangent to the boundary of the plastic region. Calculated positions of the material points on the stream lines are mapped on the monitor screen by pixels at each displacement increments $d x$.

Increase of the ellipse contact boundary from variant 3 to 1 in Table 1 and from (c) to (a) in Figure 4 results in drastic grid distortion and thickness decrease of the surface plastic layer.

Contact shear stress effect on the pressure at the points $O$ and $A$, loads $Q$ and $F$, torque $M$, surface plastic layer thickness $h_{p}$ and maximum plastic strain $e_{p}$ is given in Table 2 for the three variants of the ellipse contact boundary in Table 1.

Numerical results in Table 2 show essential effect of the ellipse contact curvature on the surface plastic flow. Contact friction decrease results in homogeneous plastic strain distribution through the surface plastic layer.

Table 1. Variants of the ellipse contact boundary.

\begin{tabular}{cccccccc}
\hline Variant & $-\omega_{O}$ & $-\omega_{A}$ & $-\theta_{O}$ & $-x_{C}$ & $y_{C}$ & $\alpha_{A}$ & $l_{c}$ \\
\hline 1 & 0.05 & 0.26 & 1.47 & 0.15 & 1.99 & 0.39 & 0.22 \\
2 & 0.50 & 0.75 & 0.74 & 0.97 & 1.54 & 0.25 & 0.36 \\
3 & 1.10 & 1.30 & 0.25 & 0.66 & 1.09 & 0.11 & 0.38 \\
\hline
\end{tabular}


Table 2. Contact friction effect on the surface plastic deformation by sliding elliptical cylinder.

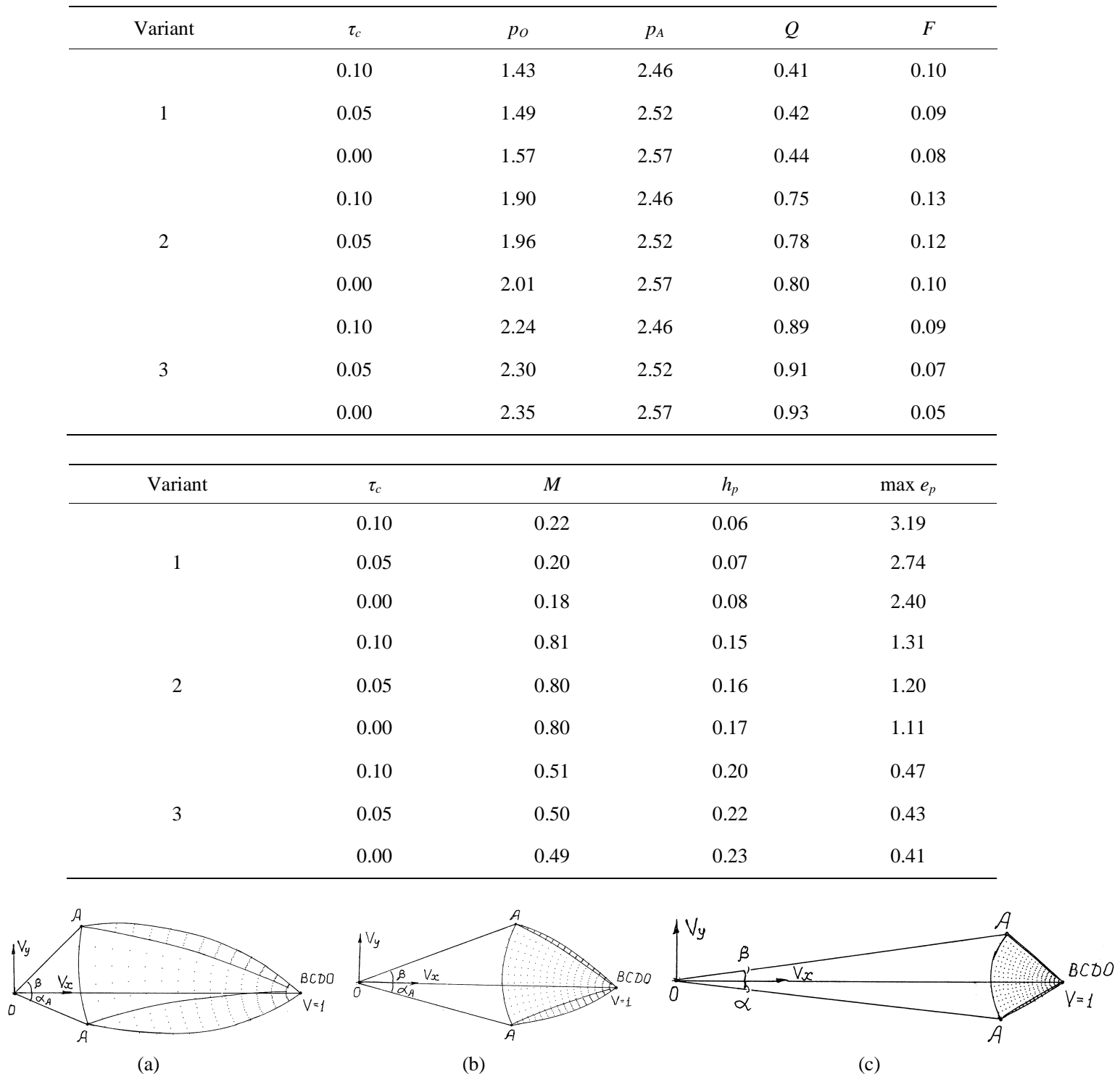

Figure 3. Velocity hodographs for the plastic regions (a), (b), (c) in Figure 2.

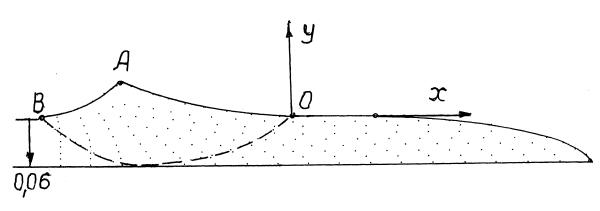

(a)

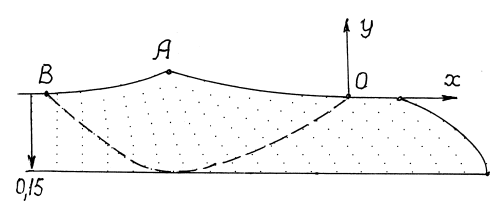

(b)

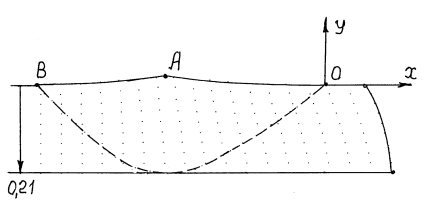

(c)

Figure 4. Stream lines with grid distortions for the plastic regions (a), (b), (c) in Figure 2.

Dimensional values of the ellipse center coordinates, contact length and plastic layer thickness are defined by multiplication of non-dimensional values on dimension value of the minor ellipse half-length $b$. Dimension shear stress $\tau_{c}$ and pressures $p$ are defined by multiplication of non-dimensional values on double plastic constant $2 k$, where $k=\sigma_{Y} / 2$ for Tresca, or $k=\sigma_{Y} / \sqrt{ } 3$ for Mises yield criterion. The material yield stress $\sigma_{Y}$ should be specified using compression stress-strain curve in the range of mean plastic strains in Figure 2 and in Table $\mathbf{2}$, to approximate material work hardening effect. 


\section{Conclusions}

Surface steady state plastic deformation by sliding elliptical cylinder with contact friction effect is modeled using hyperbolic differential equations of the plane strain ideal plasticity theory.

Plastic region with curved stress free boundary is calculated by numerical solution of nonlinear vector equation with unknown contact pressure distribution. Numerical results show essential effect of the ellipse contact boundary curvature on the mode of the surface plastic flow. Curvature of the contact boundary can be changed by rotation of the ellipse principal axes relative the surface boundary.

Numerical model is performed by FORTRAN program to investigate effects of the ellipse form, curvature and length of contact boundary and contact friction on the plastic flow of the surface with contact pressure and plastic strain distributions, loads and torque applied to the cylinder. These results, deems, are useful for engineering problems of metal parts surface plastic deformation to increase wear and fatigue resistance.

\section{Acknowledgements}

Russian Federation Ministry of Education and Science support by project No. 9.2445.2014/K is gratefully acknowledged.

\section{References}

[1] Howell, M., Hahn, G.T., Rubin, C.A. and McDowell, D.L. (1995) Finite Element Analysis of Rolling Contact for Nonlinear Kinematic Hardening Bearing Steel. Journal of Tribology, 117, 729-736. http://dx.doi.org/10.1115/1.2831544

[2] Shiratori, M., Ito, M. and Hashimoto, M. (1995) Elastic-Plastic Analysis of Rolling Contact for Surface Hardened Steel. Trans. Jap. Soc. Mech. Eng. A., 61, 1064-1069. http://dx.doi.org/10.1299/kikaia.61.1064

[3] Hill, R. (1985) The Mathematical Theory of Plasticity. 11th Edition, Oxford University Press, Oxford.

[4] Ishlinsky, A.Yu. and Ivlev, D.D. (2001) The Mathematical Theory of Plasticity. FIZMATLIT, Moscow.

[5] Marshall, E.A. (1968) Rolling Contact with Plastic Deformation. Journal of the Mechanics and Physics of Solids, 16, 243-254. http://dx.doi.org/10.1016/0022-5096(68)90032-X

[6] Collins, I.F. (1972) A Simplified Analysis of the Rolling of Cylinder on a Rigid/Perfectly Plastic Half-Space. International Journal of Mechanical Sciences, 14, 1-14. http://dx.doi.org/10.1016/0020-7403(72)90002-1

[7] Nepershin, R.I. (2002) On Rolling and Sliding of a Cylinder along a Perfectly Plastic Half-Space with Allowance for Contact Friction. Doklady Physics, 47, 256-259. http://dx.doi.org/10.1134/1.1467875

[8] Nepershin, R.I. (2003) The Rolling and Slipping of a Cylinder along the Boundary of an Ideally Plastic Half-Space. Journal of Applied Mathematics and Mechanics, 67, 293-301. http://dx.doi.org/10.1016/S0021-8928(03)90015-3

[9] Nepershin, R.I. (2013) Plastic Deformation of Surface Layer during Rigid Cylinder Rolling and Sliding. Journal of Friction and Wear, 34, 204-207. http://dx.doi.org/10.3103/S1068366613030112

[10] Nepershin, R.I. (2001) On Sliding of Obtuse Wedge along the Boundary of a Perfectly Plastic Half-Space. Doklady Physics, 46, 885- 887. http://dx.doi.org/10.1134/1.1433536

[11] Druyanov, B.A. and Nepershin, R.I. (1994) Problems of Technological Plasticity. Elsevier, Amsterdam.

[12] Dennis, J.E. and Shnabel, R.B. (1983) Numerical Methods for Unconstrained Optimization and Nonlinear Equations. Prentice-Hall, Englewood Cliffs.

[13] Broyden, C.G. (1965) A Class of Methods for Solving Nonlinear Simultaneous Equations. Maths. Comp., 19, 577-593.

[14] Nepershin, R.I. (2004) Rolling and Sliding of Rigid Cylinder along the Boundary of a Rigid-Plastic Half-Space. Mechanics of Solids, 39, 81-93. 